\title{
A Proposal for a Substrate-Assisted Catalytic Mechanism for Serine Peptidases
}

Michael N. G. James ${ }^{1}$, Jiang Yin ${ }^{1}$, Juan Colazo ${ }^{1}$, Fabricio Mosquera ${ }^{2}$, John C. Vederas ${ }^{2}$

Departments of ${ }^{1}$ Biochemistry and ${ }^{2}$ Chemistry

University of Alberta, Edmonton, Alberta, Canada, T6G2H7

Perhaps the most studied of the peptidases is the chymotrypsin family. The enzymatic mechanism involving the "catalytic triad" is firmly established in Biochemistry texts. There are parts of the acylation step, however, that remain unclear. It is well known in Physical Chemistry that $\mathrm{R}-\mathrm{OH}$ groups are poor nucleophiles, if they are nucleophilic at all. His57 has a pKa for the imidazolyl ring of $\sim 6.5$ to 7.0 ; the pKa of the Ser195 OH group is somewhere between 13.0 and 15.0, several orders of magnitude greater. So how does the Ser195 OH transfer its proton to the His57Nع2 in order for it to become nucleophilic? As far as I can tell this point has never been dealt with satisfactorily. Mike Laskowski Jr. and his coworkers have studied the interactions and inhibition kinetics of a huge number (in the hundreds) of variants of the third domain of the turkey ovomucoid bound to a panel of six serine peptidases. Members of my lab have determined the mediumresolution (and a few high-resolution) structures of many of the P1 variants of OMTKY3 bound to SGPB, Subtilisin, Chymotrypsin and Porcine Pancreatic Elastase. From all of these studies, we feel that we have an excellent view of the substrate-bound form of the serine peptidases. Close examination of these structures has led to a new hypothesis regarding the transfer of the proton on Ser195 OH to His57Nع2. Several fluorogenic substrates and substrate analogues based on this hypothesis have been synthesized and the resulting activity assays and inhibition kinetics of the substrate analogues lend strong support for the new proposal. This story forms the basis for my presentation. 